\title{
Philosophiques
}

\section{Luttes pour la reconnaissance et politique délibérative}

\section{Hervé Pourtois}

Volume 29, numéro 2, automne 2002

La démocratie délibérative

URI : https://id.erudit.org/iderudit/006256ar

DOI : https://doi.org/10.7202/006256ar

Aller au sommaire du numéro

\section{Éditeur(s)}

Société de philosophie du Québec

\section{ISSN}

0316-2923 (imprimé)

1492-1391 (numérique)

Découvrir la revue

\section{Citer cet article}

Pourtois, H. (2002). Luttes pour la reconnaissance et politique délibérative. Philosophiques, 29(2), 287-309. https://doi.org/10.7202/006256ar

\section{Résumé de l'article}

Les idées de démocratie délibérative et de politique de la reconnaissance ont été forgées en vue de répondre aux insuffisances du libéralisme politique. Les implications normatives de ces deux idées ne sont pas, ainsi qu'on le croit parfois, conflictuelles. En effet, le principe normatif sous-jacent aux demandes politiques de reconnaissance, l'éradication des sources sociales de mésestime, ne peut être honoré que par la délibération publique. Toutefois l'aptitude et la disposition des citoyens à entrer dans le processus délibératif présuppose des formes de reconnaissance prédélibératives dans le cadre de la division sociale du travail et un usage public de la raison attentif aux effets de mésestime qu'il peut engendrer. 


\title{
Luttes pour la reconnaissance et politique délibérative
}

\author{
HERVÉ POURTOIS \\ Université catholique de Louvain \\ pourtois@etes.ucl.ac.be
}

\begin{abstract}
RÉSUMÉ. - Les idées de démocratie délibérative et de politique de la reconnaissance ont été forgées en vue de répondre aux insuffisances du libéralisme politique. Les implications normatives de ces deuxidées ne sont pas, ainsi qu'on le croit parfois, conflictuelles. En effet, le principe normatif sous-jacent aux demandes politiques de reconnaissance, l'éradication des sources sociales de mésestime, ne peut être honoré que par la délibération publique. Toutefois l'aptitude et la disposition des citoyens à entrer dans le processus délibératif présuppose des formes de reconnaissance prédélibératives dans le cadre de la division sociale du travail et un usage public de la raison attentif aux effets de mésestime qu'il peut engendrer.
\end{abstract}

\begin{abstract}
The ideas of a deliberative democracy and of a politics of recognition were designed to overcome some difficulties inherent to political liberalism. The normative implications of one is not opposed to those of the other, because the normative principle underlying the political struggles for recognition - the elimination of social sources of misrecognition - has to be implemented through public deliberation. Nevertheless, the citizens' ability and motivation to take part in public deliberation depend on predeliberative forms of recognition, especially their inclusion in the social division of work. They require also that citizens pay close attention to the effects of misrecognition brought about by their public use of reason.
\end{abstract}

L'émergence récente en philosophie politique de l'idée de démocratie délibérative est à peu près contemporaine de celle de l'idée de politique de la reconnaissance ${ }^{1}$. Les deux notions sont utilisées par leurs promoteurs respectifs ${ }^{2}$ pour désigner des exigences normatives adressées au pouvoir politique. Pour les tenants de la démocratie délibérative, les procédures de formation de l'opinion et de la décision politique devraient être ouvertes à un échange discursif de raisons entre les citoyens. Pour les défenseurs de la politique de la reconnaissance, les décisions publiques devraient être sensibles aux différences culturelles et soucieuses de la protection des identités culturelles. Ces deux approches entendent ainsi répondre, chacune à sa manière, aux défis du pluralisme moral et culturel que posent nos sociétés contemporaines. La politique de la reconnaissance semble y apporter une réponse substantielle : elle met en avant un des

1. L'auteur remercie Dominique Leydet et deux lecteurs anonymes des précieuses observations qu'ils ont formulées sur des versions antérieures de ce texte. II assume évidemment seul la responsabilité des difficultés qui y subsisteraient.

2. Sur la démocratie délibérative voy. l'introduction de $D$. Leydet à ce volume et la bibliographie établie par André D uhamel (2002 : 255-274). Sur la politique de la reconnaissance voy. notamment Taylor, 1994 et Fraser, 1996 et 1998a. 


\section{8 - Philosophiques / Automne 2002}

objectifs des décisions publiques qui ne devraient pas se limiter à garantir les libertés fondamentales et la justice sociale. En revanche, la démocratie délibé rative adopte un point de vue plus procédural : elle précise la forme du processus de décision politique que l'on doit mettre en œuvre, en particulier lorsque I'on constate des désaccords moraux entre les citoyens. Cette différence de perspective explique que l'on est souvent tenté d'opposer les deux approches.

En effet, pour un défenseur d'une politique de la reconnaissance des spécificités culturelles, le concept de démocratie délibérative risque d'apparaître trop abstrait. Les minorités culturelles ne demandent pas seulement que leur discours soit entendu ou même débattu mais surtout que leurs pratiques et leurs modes de vie soient reconnus et protégés. De surcroît, la forme d'argumentation prônée par les théoriciens de la démocratie délibérative ne serait pas neutre et pourrait contribuer à la reproduction d'une domination culturelle qui opprime ces minorités ${ }^{3}$. Elle fragiliserait davantage leur position qu'elle ne l'améliore.

De l'autre côté, les tenants de la démocratie délibérative feront valoir que toute différence ou toute spécificité culturelle ne mérite pas d'être protégée. Les normes et les pratiques culturelles, qu'elles soient majoritaires ou minoritaires, doivent être éradiquées lorsqu'elles sont injustes. Et seules des procédures vraiment délibératives peuvent garantir la possibilité de leur problématisation. De plus, une politique de la reconnaissance risque de produire un compartimentage de la société en une diversité de communautés ethniques et linguistiques et de favoriser, ce faisant, un repli identitaire qui porte pré judice à l'existence d'un espace public commun et au développement d'une discussion commune authentique ${ }^{4}$.

À première vue, les implications normatives découlant de la démocratie délibérative et de la politique de la reconnaissance sont donc potentiellement conflictuelles. L'objectif du texte qui suit est de montrer non seulement que cette première impression s'avère, en grande partie, inexacte mais que démocratie délibérative et politique de la reconnaissance se présupposent mutuellement. $N$ ous essaierons d'analyser la nature de ce lien.

Dans un premier temps (1), je m'efforcerai de déjouer certains malentendus en précisant l'enjeu normatif sous-jacent à une politique de la reconnaissance : I'éradication des sources sociales de la mésestime. Le travail d'Axel $\mathrm{H}$ onneth nous aidera à clarifier le sens de cet enjeu. Cela permettra de montrer ensuite (2) qu'une démocratie délibérative s'avère nécessaire à la poursuite de cet objectif. Elle n'est toutefois pas suffisante. En effet (3) la participation à la délibération n'est possible que moyennant des conditions motivationnelles qui peuvent être affectées par les formes que prend la reconnaissance ou la non-reconnaissance de certains citoyens. Or ces conditions ne sont pas inhérentes à la pratique délibérative. $\mathrm{N}$ ous en tirerons deux considérations.

3. Sur cette critique, voy. Young, 1996 et Williams dans ce volume.

4. Sur ce type de critique, voy. M esure et R enaut, 1999 ou Todorov, 1995. 
La première considération (4), de nature structurelle, insistera sur le fait que des formes sociales de reconnaissance prédélibératives sont nécessaires à la délibération et peuvent être soutenues par un système juridique qui protège les droits fondamentaux et surtout par l'instauration d'une juste coopération sociale.

La seconde considération (5) est qu'une délibération authentique, dans laquelle les protagonistes sont mus par la recherche du meilleur argument, n'est pas à l'abri de l'expression de points de vue qui, bien que fondés sur des raisons, peuvent parfois générer de la mésestime sociale. Le problème devient particulièrement aigu lorsque le déni de reconnaissance ainsi suscité par le débat compromet la poursuite de celui-ci pour toutes les personnes intéressées. Dans leur usage public de la raison, les citoyens devraient demeurer attentifs aux effets de mésestime sociale que celui-ci peut induire.

\section{1}

11

Le vocable «politique de la reconnaissance» a été forgé par Ch. Taylor (1994) et repris par d'autres pour désigner une exigence normative adressée au pouvoir politique qui, jusqu'à présent, n'aurait pas été suffisamment prise au sérieux par les Etats démocratiques et par la pensée politique. Cette exigence serait celle que mettent en avant divers groupes et mouvements sociaux contemporains luttant pour la reconnaissance et viendrait, en quelque sorte, s'ajouter aux demandes plus traditionnelles liées à la protection des libertés fondamentales, à l'égalité des chances et à la juste distribution des ressources. On pense évidemment d'emblée aux luttes menées par des minorités culturelles en vue de faire respecter leurs particularités dans des Etats qui, bien que démocratiques, seraient encore trop «aveugles à la différence». Toutefois, si c'est bien ce type de demande que Taylor et d'autres ont en vue lorsqu'ils évoquent la politique de la reconnaissance, on doit aussi admettre que des luttes pour la reconnaissance se manifestent aussi en dehors du champ ethnoidentitaire. Elles sont aussi menées par des mouvements féministes, des minorités sexuelles ou des minorités raciales, par exemple, qui ne réclament pas la préservation d'une culture ${ }^{5}$. Et elles sont même présentes au sein de la sphère socio-économique : la reconnaissance au travail est devenue une revendication prioritaire pour certaines catégories socio-professionnelles et un objet d'investigation pour les psychologues et sociologues ${ }^{6}$. II serait donc conceptuellement inadéquat de confiner les demandes politiques de reconnaissance aux revendications ethno-identitaires. Elles sont beaucoup plus diversifiées dans leurs origines et dans leurs natures.

Si on veut appréhender adéquatement l'enjeu normatif sous-jacent à ce que pourrait être une politique de la reconnaissance, il importe d'identifier le

5. Habermas, 1998 , p. 214 ss.

6. Dejours, 1998, Sainsaulieu, 1987. 


\section{0 - Philosophiques / Automne 2002}

dénominateur commun aux différents mouvements auxquels il vient d'être fait allusion. Par delà la diversité des objets de revendications et des acteurs qui les portent, ces mouvements protestent contre le manque d'estime sociale dont serait victime un groupe. L'intuition normative qui les mobilise est que la mésestime sociale ${ }^{7}$, qui peut prendre des formes très diverses, constitue un mal moral, une injustice. Elle peut constituer une forme de dommage appelant une réponse socio-politique. C'est ce point que souligne précisément Ch. Taylor lorsqu'il écrit :

Une personne ou un groupe de personnes peuvent subir un dommage ou une déformation réelle si les gens ou la société qui les entourent leur renvoient une image limitée, avilissante ou méprisable d'eux-mêmes. La non-reconnaissance ou la reconnaissance inadéquate peuvent causer du tort et constituer une forme d'oppression, en emprisonnant certains dans une manière d'être fausse, déformée et réduite ${ }^{8}$.

Le dommage ainsi occasionné serait une injustice ${ }^{9}$ culturelle ou symbolique de nature qualitativement différente de celles qui sont occasionnées par une inégale distribution des ressources ou par une atteinte aux libertés fondamentales. Et comme les minorités ethniques ou linguistiques ne sont pas le seul type de groupe à souffrir de ce genre d'injustice, il n'y a pas lieu de considérer qu'une politique de la reconnaissance se réduirait à une politique de protection de ces minorités.

L'idée que la mésestime peut être une forme d'injustice n'est pas tout à fait neuve. D epuis très longtemps, nos systèmes juridiques ont intégré dans la notion de dommage moral le fait que certaines attitudes injurieuses ou diffamatoires peuvent porter préjudice à un individu en accréditant socialement une image peu avenante de sa personne. Dans de nombreux Etats de droit, ce type de dommage peut donner lieu à réparation et ses auteurs peuvent être sanctionnés au plan pénal. L'idée de politique de la reconnaissance va toutefois plus loin que les théories classiques du dommage moral. En effet, la mésestime prend des formes plus complexes, plus diversifiées et plus insidieuses que la calomnie ou l'injure. De plus, elle n'est pas toujours ni même d'abord imputable à un comportement individuel qui pourrait être sanctionné juridiquement. La mésestime peut être sociale lorsqu'elle est générée par des schèmes culturels ancrés dans des «habitus» et dans des institutions. Les

7. Ce terme exprime ce que la langue anglaise signifie de manière plus précise par le mot «misrecognition», c'est-à-dire non pas l'absence de reconnaissance mais la reconnaissance inadéquate.

8. Taylor, 1994, p. 41-42.

9. Certains théoriciens de la reconnaissance auraient probablement quelques réticences à caractériser la mésestime sociale d'injustice. Parfois la reconnaissance est présentée comme une exigence se situant au-delà de la justice. Je ne m'embarrasserai pas ici de ces considérations catégorielles. J e caractériserai d'injustice toute forme de dommage social infligé à un individu ou à un groupe et qui appelle une responsabilité politique en vue de l'éradiquer soit dans ses effets, soit dans ses causes. 
dénis de reconnaissance peuvent être « enracinés dans des modèles sociaux de représentation, d'interprétation et de communication ${ }^{10}$. Toute société produit en effet ce que J. Tully appelle des «normes de reconnaissance intersubjective ${ }^{11}$. Au moyen de ces normes, elle assigne à certaines catégories d'individus (parfois à l'ensemble des membres de la société en question) des qualités, des capacités, des besoins ou des dispositions qui sont présentés comme plus ou moins appréciables ou respectables. Les luttes politiques pour la reconnaissance menées par certains acteurs mettent en question de telles normes de reconnaissance dominantes lorsqu'elles sont perçues comme une source de l'humiliation et du mépris. Parfois aussi, ces luttes promeuvent des normes alternatives jusqu'alors minoritaires ou inédites. II est clair que de telles demandes ne peuvent trouver de solution par les voies judiciaires traditionnelles. Elles appellent des transformations sociales et culturelles qui doivent être, au moins partiellement, réalisées par des voies politiques. Une politique de la reconnaissance serait donc une politique qui viserait à transformer les conditions sociales qui génèrent cette forme d'injustice qu'est la mésestime sociale frappant un groupe ou des individus ou, pour le dire en d'autres termes, à éradiquer les normes de reconnaissance inadéquates pour les remplacer par des normes plus adéquates.

Une telle caractérisation demeure purement formelle. Elle ne réfère pas, comme on le fait trop souvent, la politique de la reconnaissance à un type particulier d'action collective ou d'intervention publique circonscrit au domaine spécifique de la protection des identités culturelles. Elle ne la réduit pas non plus à une «politique de la différence» que l'on opposerait à des politiques uniformisantes. Comme le suggère $\mathrm{N}$. Fraser, la question de savoir si une politique de la reconnaissance implique un traitement différencié de certains groupes ou, au contraire, un traitement identique de tous les citoyens appelle une réponse qui ne peut être que pragmatique ou empirique : cela dépend du type de mésestime sociale auquel on essaie de remédier, du type de norme de reconnaissance qui est mis en question ${ }^{12}$. Ce serait donc faire un mauvais procès à l'idée de politique de la reconnaissance que de la condamner au nom des risques de sacralisation de la différence. $C$ ar ce qui est en jeu n'est pas un très hypothétique «droit à la différence» mais plutôt un droit à mettre en question des stéréotypes culturels qui génèrent le mépris et I'humiliation.

$\mathrm{N}$ otons d'ailleurs que notre caractérisation ne répond pas non plus à la question de savoir ce qui dans la mésestime sociale est facteur d'injustice. Pour certains, elle serait une injustice parce qu'elle priverait ceux qui en sont victimes de droits fondamentaux (par exemple, le droit de vote) ou de l'accès aux bénéfices des dispositifs de redistribution socio-économique ${ }^{13}$. Pour

10. Fraser, 1995a, p. 71.

11. Tully, 2000, p. 471.

12. Fraser, 1998a et b.

13. Young, 1997. 


\section{2 - Philosophiques / Automne 2002}

d'autres, la mésestime sociale constituerait une injustice en soi, en raison du mépris qu'elle génère et cela indépendamment des effets dommageables qu'elle occasionne sur le plan redistributifit ${ }^{14}$. Comme cela ne me paraît pas nécessaire à la suite de notre propos, je ne me prononcerai pas ici sur l'approche qu'il faut privilégier .

Une question mérite toutefois d'être traitée. Pourquoi la mésestime sociale constitue-t-elle un dommage qui pose problème sur le plan moral? La réponse la plus élaborée à cette question a été récemment apportée par le philosophe allemand Axel $\mathrm{H}$ onneth ${ }^{15}$.

\section{2.}

Dans Kampf um Anerkennung, Axel $\mathrm{H}$ onneth entreprend une reconstruction de la « grammaire morale des conflits sociaux ». La thèse développée est que tout conflit social est sous-tendu par une lutte pour la reconnaissance qui lui donne une signification morale. En effet, l'enjeu d'un conflit social n'est pas seulement l'accès au pouvoir ou à des ressources mais le statut et l'image qui sont publiquement assignés à une personne ou à un groupe. Lorsque les structures sociales ou les modèles culturels en vigueur induisent des images et des statuts dépréciatifs pour certains groupes, cela peut engendrer chez ceux qui en sont victimes une difficulté, voire une impossibilité, à construire un rapport positif à soi. A lors surgit un problème moral dans la mesure où les questions morales se rapportent, selon $\mathrm{H}$ onneth, aux dispositifs à mettre en place pour protéger la vulnérabilité de l'individu dans son aptitude à développer un rapport positif à soi ${ }^{16}$.

À partir d'une analyse fine des expériences du mépris vécu comme déni de reconnaissance, $\mathrm{H}$ onneth distingue trois formes de reconnaissance irréductibles : la bienveillance, le respect et l'estime sociale ${ }^{17}$ (ou solidarité) qui sont respectivement structurantes pour les trois modalités fondamentales du rapport à soi : la confiance en soi, le respect de soi et l'estime de soi. J en'évoquerai ici que les deux dernières dimensions. Car elles sont, à la différence de la première, directement pertinentes sur le plan politique ${ }^{18}$.

Une des figures du mépris réside dans ces attitudes qui manifestent qu'une personne n'est pas reconnue dans son autonomie, c'est-à-dire comme un être capable de discerner ce qui est bon/juste et d'agir en conséquence. Le

14. Fraser, 1998a.

15. H onneth, Axel, 1992, 1996, 1998.

16. I bid., 1996, p. 1276.

17. Le terme «estime sociale» reçoit ici un sens plus étroit que celui auquel je me suis référé plus haut lorsque j'ai introduit la notion de mésestime sociale (misrecognition). Celle-ci couvre globalement les manques de reconnaissance dans les trois dimensions identifiées par H onneth : la bienveillance, le respect et l'estime sociale. Cette dernière désigne, pour lui, la reconnaissance de la valeur des aptitudes et caractéristiques particulières d'un individu ou d'un groupe.

18. J'écris «semblent» parce que il serait possible de montrer que l'assurance d'une confiance en soi a aussi une dimension politique y compris dans l'espace de la délibération. Je ne puis toutefois développer ici une telle thèse. 
fait d'être privé de droits fondamentaux ou d'être exclu d'une communauté politique peut occasionner ce type de déni de reconnaissance. II contribue à miner le respect de soi qui repose sur la conscience d'être capable d'interagir avec d'autres puisque l'on a la faculté de juger des normes de la coexistence sociale. À l'inverse, ce respect de soi se trouvera conforté par le respect mutuel, qui est cette attitude par laquelle on se rapporte à l'autre comme à un être avec lequel il est possible d'interagir sur un pied d'égalité dans la mesure où il est capable de jugement pratique, et peut, pour cetteraison, contribuer à la définition commune des normes sociales.

Une autre figure du mépris distinguée par $\mathrm{H}$ onneth se marque dans des attitudes ou des normes institutionnelles ou culturelles qui manifestent que la contribution particulière d'une personne (ou d'un groupe) à la vie sociale est dépréciée ou ignorée. Q uand l'apport ou les aptitudes particulières d'une personne sont niés, sous-estimés, discrédités de manière systématique, alors celleci peut souffrir d'un déficit d'estime de soi qui l'empêche de développer une image positive de son mode de vie singulier et de ses aptitudes spécifiques. À l'inverse, l'estime sociale que nous portons à des particularités bonnes et précieuses peut être source d'estime de soi pour ceux qui les cultivent. Un impératif moral conceptuellement différent de celui qui impose le respect trouve ici son origine : il s'agit d'assurer à chacun les opportunités réelles d'accéder à l'estime sociale, en apportant sa contribution à la vie sociale et en faisant en sorte que celle-ci soit reconnue. Une juste répartition et une juste rétribution du travail sont certainement fondamentales de ce point de vue.

\section{2}

2.1.

Ce n'est pas sans raison que la réflexion actuelle sur la reconnaissance est contemporaine d'un réexamen de la théorie normative de la démocratie qui a propulsé à l'avant-plan l'idée de démocratie délibérative ${ }^{19}$. En effet, l'émergence des conceptions délibératives procède, elle aussi, d'une insatisfaction à l'égard des théories de la justice qui se sont développées à la suite de celle de Rawls et cela, tant dans le camp libertarien que dans le camp égalitariste. L'objectif des tenants de la démocratie délibérative est de dépasser ou, en tout cas, de prolonger le débat sur les principes de justice par une réflexion sur les processus de formation de l'opinion et de la décision politique. II s'agit d'identifier les conditions que doivent satisfaire ces processus pour que les exigences morales (en particulier les exigences de justice) puissent être prises en considération et réalisées dans la sphère politique. La condition majeure réside dans le fait que ces processus devraient prendre la forme d'une discussion argumentée ouverte à tous les points de vue et orientée vers la production d'un accord raisonné. Une société est démocratique lorsque «ses

19. Les références sur le sujet sont nombreuses. Voir la bibliographie établie par André Duhamel (2002: 255-274). 


\section{4 - Philosophiques / Automne 2002}

affaires sont gouvernées par la délibération publique de ses membres ${ }^{20}$. Une caractérisation nouvelle de l'idéal démocratique est donc proposée. Celui-ci ne peut se dissoudre dans l'idée classique de la souveraineté du peuple, futelle conjuguée au concept tout aussi classique de l'Etat de droit. La démocratie ne peut être identifiée à des mécanismes d'agrégation des préférences conflictuelles sanctionnés par la règle majoritaire et limités par un contrôle judiciaire du respect de la Constitution et des libertés fondamentales. Elle présuppose plus fondamentalement que les décisions prises par les autorités publiques résultent de procédures d'échanges de raisons dans lesquelles toute partie concernée doit pouvoir faire valoir et soumettre les siennes.

Plusieurs arguments ont été invoqués en faveur d'un tel modèl $\mathrm{e}^{21}$. Le plus fort de ceux-ci est qu'une telle approche de la démocratie permet de dépasser la conception privatiste étroite de la liberté présente dans la pensée politique libérale. L'autonomie de l'individu ne peut être pleinement garantie que lorsque le citoyen prend part à l'élaboration discursive de la loi qui s'impose à tous.

\section{2 .}

Une des thèses soutenues par les défenseurs d'une démocratie délibérative, en particulier $\mathrm{H}$ abermas, est que celle-ci permettrait de rencontrer les attentes de reconnaissance formulées par de nombreux groupes sociaux ${ }^{22}$. Compte tenu de la manière dont nous avons défini la politique de la reconnaissance, nous pouvons nous rallier à cette thèse. Une délibération publique authentique devrait mener à l'instauration de justes normes de reconnaissance, ou en tout cas de normes de reconnaissance moins injustes. On pourrait même ajouter que davantage encore que tout autre conflit, les luttes pour la reconnaissance demandent une résolution par le débat. $C$ ar ce qui est en question, ce n'est pas d'abord le partage du pouvoir, de l'influence ou des ressources. L'enjeu explicite pour les acteurs en lutte est une norme culturelle susceptible d'induire de la mésestime sociale - par exemple, la norme qui hiérarchise les formes de contribution à la vie sociale sur l'opposition travail domestique/travail salarié. Dans la mesure où au moins un des acteurs donne une telle signification morale à sa lutte, en problématisant la justesse d'une norme culturelle en vigueur et pas seulement des intérêts ou des rapports de force, la résolution politique du conflit ne peut passer que par un processus qui soumet à l'échange des raisons les prétentions normatives en présence. Certes, il arrive que certains groupes minorisés ou dominés (ou se disant tels) usent de manière rhétorique de la plainte morale et que ce qu'ils présentent comme une lutte pour la reconnaissance ne soit en fait que l'expression dissimulée d'intérêts pour l'appropriation du pouvoir ou d'avantages matériels. M ais cela ne fait que justifier davantage encore le recours à la délibération dans la mesure où elle permettra de discriminer les raisons

20. Cohen, 1989, p. 17.

21. Cooke, 2000.

22. H abermas, 1998, p. 205-244. 
normatives fortes liées à la reconnaissance par rapport aux motifs liés à des intérêts particuliers (qui ne sont d'ailleurs pas pour autant des motifs illégitimes de protestation). La délibération peut ainsi, sinon résoudre, à tout le moins élucider la source normative éventuelle d'un conflit social.

De plus, la généralisation de dispositifs de délibération publique favorise l'expression des points de vue sur les normes de reconnaissance en vigueur, en particulier les points de vue de ceux qui en sont victimes ou pensent l'être. Cette expression, soumise à l'épreuve du débat, devrait contribuer à une « interprétation adéquate ${ }^{23}$ des besoins spécifiques et à une prise en considération des particularités culturelles de chaque groupe. Elle est le prélude à un échange sur les raisons qui fondent les normes en vigueur et les normes alternatives mises en avant par tel ou tel groupe social.

Cet échange peut parfois conduire à la production de normes différenciées, à l'octroi de statuts ou de droits spécifiques pour certains groupes dans I'espace politique commun. La délibération publique exige certes une stricte égal ité dans l'accès au débat public et une impartialité dans le traitement des questions. M ais elle autorise des traitements différenciés au niveau substantiel lorsque ceux-ci sont nécessaires, par exemple, pour protéger une culture menacée ou pour réhabiliter des activités dévalorisées en raison même du fait qu'elles étaient exercées par des femmes. La délibération publique permet une attention croissante au contexte qui n'implique ni le refus ni la sacralisation de la différence. $C$ ar, tout en étant ouvert aux prétentions particulières élevées par les acteurs, le débat public peut aussi les problématiser et en poser les limites. Un échange de raisons peut ainsi déboucher sur la conclusion que même si la plainte élevée par une minorité culturelle est légitime, elle ne justifie que peu ou pas de mesures de protection spéciales car celles-ci mettraient en danger la liberté des membres à l'égard de l'emprise du groupe minoritaire. Le débat public favorise la mise en question des normes de reconnaissance qui généraient I'humiliation et le mépris et la construction de nouvelles normes qui surmontent le déni ainsi vécu.

L'aptitude à intégrer et à traiter les demandes substantielles de reconnaissance marque incontestablement la supériorité du modèle délibératif de la démocratie sur le modèle libéral classique pour lequel le champ politique est celui du compromis entre des préférences conflictuelles non discutées. Cette dernière approche ne laisse finalement guère d'espace à la transformation des normes culturelles dominantes face à des normes minoritaires ou alternatives car ces normes tendent à être assimilées précisément à de simples préférences. En revanche, une politique délibérative serait à la fois plus sensible aux demandes morales des acteurs que ne peut l'être une instance juridictionnelle statuant sur des droits et moins encline à entériner les préférences, les rapports de force et les normes en vigueur comme tend à le faire la simple négociation d'un compromis.

23. Ibid., p. 212-213. 


\section{6 - Philosophiques / Automne 2002}

2.3.

Le lien interne qui vient d'être posé entre politique délibérative et politique de la reconnaissance ne va pourtant pas de soi. Une des objections majeures adressées au modèle délibératif concerne ses limites cognitives internes. A l'inverse de ce que semble suggérer la lecture quelque peu idéaliste de $\mathrm{H}$ abermas ou d'autres tenants du modèle délibératif ${ }^{24}$, une délibération authentique, même correctement conduite, ne conduit que très rarement et seulement de manière fortuite à produire un accord sur des bonnes raisons normatives ${ }^{25}$. Elle ne parvient qu'exceptionnellement à résorber les désaccords raisonnables sur le juste et l'injuste et, en particulier, sur les normes d'une juste reconnaissance ${ }^{26}$. Certes, l'échange d'arguments peut contribuer à déconstruire des normes sociales génératrices de mépris. $M$ ais il conduit rarement à la production de normes alternatives unanimement admises et exemptes de reproches au regard des effets de mésestime qu'elles sont susceptibles d'engendrer. Les débats contemporains sur le statut social des prostituées ou sur le statut de minorités culturelles et linguistiques en sont de bons exemples. Les réponses proposées à des demandes de reconnaissance feront rarement l'objet d'un consensus. Toute demande suscitera une contre-demande et très souvent la réponse apportée à une demande conduira à en négliger voire à en nier d'autres ${ }^{27}$.

A insi dans le débat contemporain sur la prostitution, particulièrement vif dans certains pays européens, les positions néo-abolitionnistes défendent la pénalisation du client et du proxénète au nom de l'éradication du mépris dont est victime la femme. 0 r, même si elle ne vise pas directement la prostituée, une telle pratique conduit de fait à méconnaître non seulement sa libre disposition de soi mais aussi ses difficultés socio-économiques vécues clandestinement ou encore sa contribution, notamment fiscale, à la vie économique. À l'inverse, la perspective néo-réglementariste mise en œuvre, par exemple, aux Pays-Bas, propose d'accorder un statut professionnel à la prostituée en vue d'améliorer ses conditions de travail et de vie ainsi que de reconnaître sa contribution à la vie socio-économique tout en luttant contre la prostitution forcée. La régulation d'une activité permet d'éviter un certain nombre de dérives et de risques pour celles qui la pratiquent. $M$ ais la faiblesse d'une telle position est qu'elle contribue à occulter la violence psychique et symbolique induite par le rapport prostitutionnel et critiquée par nombre de féministes ${ }^{28}$. Le néo-réglementarisme contribuerait, lui aussi, à la reproduction de normes de reconnaissance contestées.

Dans ce débat, comme dans beaucoup d'autres domaines, il ne semble pas y avoir de solution définitive et univoque aux luttes pour la reconnaissance. Ces limites cognitives internes à la délibération pourraient conduire à conclure

24. Gutmann et Thompson 1997.

25. Weinstock, 2000, p. 419.

26. Tully, 2000, p. 472.

27. Tully, 2000, p. 476.

28. Sur ce débat voir Vielle 2001 qui défend une position néo-réglementariste. 
qu'au mieux elle est inapte à résoudre les conflits de reconnaissance et qu'au pire, elle peut contribuer à nous détourner de dispositifs de résolution alternatifs plus opératoires et moins coûteux, tels la négociation de compromis.

\section{4 .}

Face à I'objection qui précède, on peut toutefois répondre que, même si la délibération publique ne produit pas des normes substantielles de reconnaissance unanimement admises et si les solutions trouvées sont toujours provisoires, hybrides et contestées, il n'en reste pas moins que la participation à cette délibération peut induire de la reconnaissance. Le fait que la société offre au citoyen des opportunités réelles de prendre part au débat public, dans la société civile ou dans des procédures institutionnalisées, pourrait renforcer son autonomi $\mathrm{e}^{29}$, contribuer au développement de son respect de soi et de son estime de soi et accroître son sentiment d'appartenance et d'identification à la communauté politique ${ }^{30}$. Les dispositifs et procédures par lesquels les citoyens et les groupes peuvent échanger des raisons relatives aux normes de redistribution et de reconnaissance contribuent, même lorsqu'elles ne débouchent pas sur un accord, à signifier publiquement à tous que chaque citoyen doit être considéré comme capable de jugement et de discernement, doit être reconnu comme autonome. C'est d'ailleurs là, s'il faut en croire $\mathrm{H}$ abermas ${ }^{31}$, une des vertus essentielles de la discussion publique.

Sur un plan purement procédural, l'ouverture d'espaces de débat public à ceux qui luttent et qui souffrent réduirait les sources de mésestime sociale dont ils sont victimes. En effet, indépendamment du traitement réservé sur le fond aux demandes exprimées par des groupes, le seul fait de leur donner l'opportunité réelle de se manifester dans l'espace public signifierait aux yeux de tous leur aptitude d'agent moral et de citoyen.

\section{3}

Les lignes qui précèdent soulignent combien une démocratie délibérative est nécessaire à une politique d'élimination, fut-elle toujours partielle, des sources sociales de la mésestime. Les pages qui suivent tenteront d'examiner les limites de cette thèse. La théorie développée par Axel H onneth est, à cet égard, précieuse. Elle peut nous servir à proposer une seconde thèse, non pas alternative mais complémentaire, que les tenants de la démocratie délibérative ne prennent pas suffisamment au sérieux. Une politique délibérative présuppose des conditions de reconnaissance qui confèrent au citoyen la possibilité de développer un rapport positif à soi dans les dimensions mentionnées par $\mathrm{H}_{\text {onneth }}{ }^{32}$ mais qui ne peuvent être produites par la pratique délibérative elle-même.

29. Weinstock, 2000, p. 420.

30. Tully, 2000, p. 480.

31. H abermas, 1993, p. 222 ss.

32. La place manque ici pour examiner dans quelle mesure la bienveillance affective, qui est génératrice de confiance en soi, serait, elle aussi, un des présupposés politiquement signifiants de la délibération publique. 


\section{8 - Philosophiques / Automne 2002}

L'effectuation de l'idéal délibératif repose évidemment sur des mécanismes institutionnels qui ouvrent les procédures de décision publique à l'échange des raisons. Q uelles innovations institutionnelles devraient être mises en place, c'est là une question qui reste largement débattue. Les dispositifs classiques de l'Etat de droit sont-ils suffisants, comme le suggère $\mathrm{H}$ abermas? O u faudrait-il les compléter, voire les remplacer, par de nouveaux types de procédures de formation de l'opinion et de la décision publique telles que les sondages délibératifs ou les conférences de citoyens ${ }^{33}$ (Fishkin 1995)? La question n'est pas simple et n'est pas ici notre propos. M ais il est, en tout cas, évident que les mécanismes institutionnels, quelle qu'en soit la forme, seront insuffisants et, pour ainsi dire, inopérants si les citoyens ne cultivent pas certaines dispositions, certaines vertus. C'est là un point sur lequel s'accordent les théoriciens de la démocratie délibérative lorsqu'ils mettent en avant l'importance d'un patriotisme constitutionnel ${ }^{34}$ ou des vertus civiques ${ }^{35}$. Une démocratie délibérative ne suppose pas seulement des citoyens compétents capables de justifier et de discuter publiquement des normes. Elle demande aussi des citoyens qui soient disposés à entrer dans le débat public lorsqu'ils sont interpellés et à en accepter l'issue, en ce compris lorsque l'accord étant impossible, il faut opérer un compromis. L'autonomie politique des citoyens réside à la fois dans la capacité à poser un jugement politique et dans leur disposition à le faire, à en rendre raison et à accepter l'issue d'une délibération ${ }^{36}$.

Si cette thèse est assez largement partagée, il n'en reste pas moins que les réflexions sur les vertus qu'exige la participation à la délibération publique restent souvent assez vagues et, surtout, ne prennent pas suffisamment au sérieux les conditions sociales de leur épanouissement. Elles semblent en appeler à une forme d'engagement moral qui paraît déconnecté de la réalité sociale contemporaine. On pourrait en quelque sorte leur renvoyer l'objection que $\mathrm{H}$ egel adressait à la doctrine kantienne de la vertu. Le formalisme abstrait de l'impératif catégorique, en dissociant radicalement l'intérêt pur pour la raison des intérêts empiriques, prive la morale de l'ancrage motivationnel sans lequel elle ne peut s'incarner dans des pratiques individuelles et collectives. Pour $\mathrm{H}$ egel, la moralité individuelle n'est possible que moyennant l'inscription de la conscience et de la pratique dans une forme de vie éthique partagée qui s'incarne dans des mœurs et des institutions communes. De manière anal ogue, la procédure délibérative d'échange de raisons n'apporterait pas, par ellemême, les ressorts incitant les citoyens à y entrer et à reconnaître la force normative des décisions qui en émanent. O n doit admettre, en revanche, que la capacité et surtout la disposition à participer à la vie politique et sociale exigent un rapport positif à soi et que celui-ci, comme le souligne Honneth,

33. Fishkin, 1995.

34. H abermas, 1997, p. 67

35. Gutmann et Thompson, 1997, Pamental, 1999.

36. C'est que M. Cooke (1992) appelle de son côtél'autonomie comme « rational accountability». 
dépend, à son tour, de formes de reconnaissance sociale qui ne se nouent pas exclusivement dans l'espace de la discussion publique.

Dans ce qui suit, nous tenterons d'expliciter cette thèse et d'en montrer les conséquences à deux niveaux : au niveau des conditions structurelles de reconnaissance que suppose l'engagement dans le débat public (4) et au niveau de l'usage public de la raison au sein même des procédures de délibération (5).

\section{4}

4.1

$\mathrm{H}$ abermas, comme d'autres auteurs, insiste sur le fait que le patriotisme constitutionnel et les vertus délibératives ne peuvent se développer que s'ils sont nourris par une culture politique partagée des droits de l'homme et de la démocratie ${ }^{37}$. Et il faut pour cela, ajoute-t-il, que cette culture politique soit suffisamment ancrée dans I'histoire particulière de la nation, dans « des contextes de vie intersubjectivement partagés» qui donnent, en quelque sorte, à cette culture politique sa "coloration éthique» 38 et qui en font ainsi un objet possible d'identification pour les citoyens. Cette position se heurte à une limite, que $\mathrm{H}$ abermas reconnaît lui-même. Si la coloration nationale d'une culture politique est trop forte, alors les principes universels et le respect de la diversité culturelle sont menacés. En revanche, si la coloration nationale est trop faible, alors la culture politique demeure trop abstraite et perd son potentiel intégrateur.

Ce dilemme peut toutefois être dépassé, c'est le point de vue défendu ici, lorsqu'on perçoit que l'ancrage motivationnel de la démocratie délibérative ne doit pas seulement ni même d'abord être cherché dans l'identification à une nation civique mais dans l'instauration, au sein d'une communauté politique particulière, de formes sociales de reconnaissance génératrices de respect de soi et d'estime de soi. À cet égard, il ne faudrait pas négliger le potentiel de reconnaissance inclus dans un système juridique protégeant les droits fondamentaux et surtout dans une juste division sociale du travail. Dans ce qui suit, nous essaierons de suggérer que ces deux facteurs sociaux structurels de reconnaissance sont importants pour une démocratie délibérative.

\section{2 .}

S'engager dans une délibération publique n'a de sens que si l'on se perçoit soimême comme un agent moral capable de poser un jugement autonome et disposé à en rendre raison. L'accès au respect de soi, au sens défini plus haut, est donc fondamental. Cette forme du rapport positif à soi peut bien entendu être générée par la participation à la délibération publique, à des discussions dans lesquelles nos concitoyens nous reconnaissent comme interlocuteurs valables. Toutefois, dans nos sociétés complexes, les possibilités effectives

37. H abermas, 1998, p. 67 ss. et p. 215 ss.

38. I bid., p. 219. 


\section{$300 \cdot$ Philosophiques / Automne 2002}

pour un citoyen d'entrer directement - c'est-à-dire sans passer par la médiation d'organisations représentatives - dans des débats sont, somme toute, limitées. Certes on devrait espérer qu'une forme de démocratie plus délibérative offrirait davantage d'opportunités de participation directe tant à l'intérieur des espaces publics de la société civile qu'au sein des dispositifs institués de consultation, de concertation et de décision. M ais, ces occasions offertes au citoyen pris individuellement resteront rares et contingentes. II n'est d'ailleurs pas sûr que leur multiplication soit le facteur le plus déterminant d'accroissement du caractère délibératif des procédures de décision collective. C'est pourquoi l'assurance du respect de soi ne peut être offerte de manière durable par ces seules procédures. Elle présuppose aussi un système de droits fondamentaux. La garantie juridique des droits fondamentaux (en ce compris de droits socio-économiques) vise bien entendu à donner à chaque citoyen la capacité effective de s'inscrire dans le débat public. Elle marque aussi que la liberté individuelle du citoyen n'est pas à la disposition de la volonté majoritaire. $\mathrm{M}$ ais l'affirmation et la protection des droits vise en outre, de manière en quelque sorte indirecte, à signifier au citoyen l'assurance publique qu'il est capable de prendre part au jeu démocratique, parce qu'il dispose des compétences nécessaires en matière de jugement pour ce faire. Ainsi, l'octroi du droit de vote offre une possibilité effective de participation à la vie démocratique. M ais, dans une société de masse, il vise aussi surtout oserions-nous dire - à signifier publiquement que celui qui est titulaire de ce droit est capable de poser un jugement autonome. À l'inverse, celui qui en est privé n'est pas seulement empêché de participer pleinement au processus politique. II lui est aussi, par là même, dénié l'aptitude à interagir avec les autres sur un pied d'égalité dans les relations sociales ${ }^{39}$. Ceci permet de comprendre pourquoi posséder un droit à la participation, en particulier le droit de vote, est précieux même si on ne l'exerce pas et si l'on ne retire guère de bénéfice de son exercice. C'est en tout cas une des raisons pour lesquelles la garantie des droits individuels, et même parfois de droits spécifiques pour les minorités ${ }^{40}$ est précieuse pour la démocratie. Elle est non seulement une condition institutionnelle, comme le souligne $\mathrm{H}$ abermas, mais aussi une condition motivationnelle. L'inclusion dans un espace de reconnaissance que signifie le droit peut être une source fondamentale de ce respect de soi qu'exige la participation à la vie démocratique.

\section{3.}

Cette reconnaissance produite par le droit demeure toutefois insuffisante. Entrer dans un débat public sur les normes (de reconnaissance et de distribution) n'a de sens que pour celui qui s'estime capable de prendre part à la

39. Shklar, 1995.

40. Will Kymlicka, 1995, 173 ss., souligne que les demandes de droit spécifiques formulées par des minorités peuvent aussi manifester un souhait d'intégration plus forte à la communauté politique. 
coopération sociale. $O r$, cette estime de soi, relative aux aptitudes bonnes et précieuses que possède un sujet ou un groupe, ne peut se développer que là où règnent les bases de cette forme de reconnaissance que $\mathrm{H}$ onneth appelle « estime sociale». Celles-ci apparaissent donc requises pour une délibération démocratique authentique. Tel est le sens de la thèse que semble développer A. H onneth lui-même dans un article récent sans toutefois la relier explicitement à sa théorie de la reconnaissance ${ }^{41}$. C'est ce que nous allons tenter de faire.

A travers une relecture de Dewey, $\mathrm{H}$ onneth entend montrer que la résolution discursive des problèmes politiques présuppose une reconnaissance prédiscursive dans le cadre d'une coopération sociale. O n doit admettre que les procédures de délibération collective sont «l'outil cognitif au travers duquel la société s'efforce, par des voies expérimentales, d'explorer, traiter et résoudre ses problèmes propres à la coordination de l'action sociale ${ }^{42}$. $M$ ais cette justification purement épistémologique d'une forme de démocratie délibérative ne suffit pas à lui donner une valeur éthique aux yeux des citoyens. Et la conscience même de cette valeur semble difficile à développer dans des sociétés complexes où il apparaît impossible d'impliquer directement tous les citoyens dans les procédures démocratiques. C'est pourquoi le développement des procédures démocratiques présuppose une forme d'intégration sociale prédiscursive par une « conscience collective de l'association prépolitique de tous les citoyens». Celle-ci ne peut être assurée par l'identification à une nation qui contient toujours une part d'exclusion arbitraire liée aux circonstances historiques. Elle repose plutôt sur la conscience de la nécessité d'une participation coopérative et ne peut par conséquent surgir que grâce à une forme juste et équitable de la division sociale du travail (au sens large).

En l'absence de justice, non seulement dans la rétribution du travail, mais également dans sa répartition, il n'y pas d'émergence possible de cette conscience de la coopération sociale qui permet à chaque membre de la société de fonder ses orientations dans des procédures démocratiques, car c'est dans cette articulation que réside la perception de leur utilité en tant qu'instrument de résolution rationnelle des problèmes communs ${ }^{43}$.

Ceci signifie donc que l'exercice même de la délibération publique n'est possible que moyennant l'existence d'un espace de reconnaissance prédiscursif dans une sphère sociale « vécue comme coopération » .

$\mathrm{Q}$ ue devons-nous en conclure pour la question qui nous occupe? Si une politique délibérative est une condition nécessaire d'une restauration de la reconnaissance là où a prévalu I'humiliation, le mépris et la mésestime sociale, elle n'est pas une condition suffisante. Car elle présuppose non seulement la garantie de droits fondamentaux mais surtout une forme de coopération

41. H onneth, 1999b.

42. H onneth, 1999b, p. 175.

43. H onneth, 1999b, p. 176. 
sociale, c'est-à-dire d'activité dont tous les agents sont aussi les bénéficiaires. Celle-ci est génératrice d'un sens de la coopération et d'une estime de soi pour ceux qui y prennent part et, pour cette raison, donne, à leurs yeux, une raison d'être à la délibération publique.

O n objectera bien sûr que la forme que doit prendre la coopération sociale, que les critères de ce qui constitue une bonne contribution et une juste coopération font l'objet de désaccords. Par conséquent, en dernier ressort, ce n'est qu'à travers la délibération publique que l'on pourra établir si et comment un mode déterminé de coopération sociale peut être générateur de reconnaissance. Dans le champ de la division du travail, comme dans d'autres, la délibération permet la problématisation des normes de reconnaissance en vigueur dans ce qu'elles peuvent avoir d'humiliant et la construction, toujours fragile certes, de normes alternatives. Une juste coopération sociale présuppose donc une juste délibération. $M$ ais la proposition inverse est tout aussi vraie : une juste délibération publique présuppose, comme condition d'effectuation, une coopération sociale qui, à défaut d'être juste, devrait, à tout le moins, être décente au sens que $A$. M argalit donne à ce terme ${ }^{44}$. Une coopération décente ne devrait pas générer cette humiliation, cette mésestime sociale, qui affectant le rapport à soi, empêcherait un agent de se percevoir comme pleinement participant à la vie sociale. L'exclusion socio-économique, qui, on le sait, ne se réduit pas simplement à la privation du bénéfice des droits sociaux et qui s'accompagne souvent d'une vulnérabilité socio-affective ${ }^{45}$, peut, à cet égard, être considérée comme l'obstacle majeur à la décence que requiert la promotion de l'idéal délibératif.

Une communauté politique ne peut offrir les bases d'une reconnaissance mutuelle, d'une estime mutuelle, que si elle donne à tous ses citoyens l'opportunité de faire des choses ensemble, de s'inscrire dans des projets coopératifs qui les transcendent. Cette nécessité d'un agir commun avait déjà été soulignée par $H$. A rendt en son temps. M ais sa nostalgie à l'égard de la polis et sa conception réductrice du travail l'avaient menée à croire que l'action commune ne pouvait s'inscrire que dans l'espace politique. $O r$, il n'y pas de raison particulière de penser que le sens de la coopération ne pourrait se développer dans d'autres champs de la structure de base de la société : dans le champ socio-économique - où le sens de la coopération peut s'enraciner dans un projet de construction d'un système de protection sociale, par exemple - ou dans le champ de la société civil ${ }^{46}$. Sans la conviction partagée que nous avons à contribuer et à recevoir dans la réalisation de projets de coopération, l'espace public de la délibération perd sa substance et sa raison d'être ${ }^{47}$.

44. M argalit, 1996.

45. Castel, 1995.

46. Voir, par exemple, les thèses développées par M. Walzer, 1992.

47. On pourrait objecter que la conception forte de la coopération sociale ici suggérée nous expose aux critiques souvent adressées aux visions communautariennes de la communauté politique. Dans nos sociétés pluralistes, il serait impossible de s'accorder sur un projet politique 
La faiblesse des réflexions contemporaines sur la démocratie délibérative est qu'elles ne thématisent pas cette condition et semblent tenir pour acquises les formes d'organisation de la production sociale générées par le marché et, secondairement, par la régulation bureaucratique. Au vu des évolutions politiques récentes, il ne serait certainement pas superflu de se poser la question de savoir si la pratique démocratique n'exigerait pas une transformation de ces formes de coopération et surtout une éradication du monopole qu'elles ont acquis, sinon dans les faits, au moins au niveau idéologique dans la définition de ce qu'est une activité socialement utile. En d'autres termes, il y aurait lieu de penser les conditions de la cooriginarité d'une démocratie délibérative et d'une coopération sociale juste (au moins décente). Dans quelle mesure la division sociale du travail instaure-t-elle des formes de reconnaissance favorables à la délibération? Voilà une question qui mérite d'être posée, même si elle ne peut être résolue politiquement que par la délibération elle-même. A défaut, toute procédure de délibération politique demeurera abstraite ou - pire - risquera d'apparaître comme une ruse destinée à conforter la position de ceux qui profitent de l'exclusion et de l'exploitation dans le champ socio-économique et de l'atomisation des rapports sociaux.

commun. Et, de plus, nous devrions reconnaître que là où de tels projets ont vu le jour ce fut toujours au prix des nationalismes excluants et violents (Kymlicka, 1990, p. 225). À cette double objection, nous pouvons rapidement apporter une double réponse.

$1^{\circ} \mathrm{La}$ conscience partagée que nous avons à faire des choses en commun ne requiert pas un accord sur une conception de la vie bonne ni même sur une conception de la justice qui apporterait une justification substantielle à ce projet. La construction d'un système de protection sociale peut apparaître aux yeux des citoyens comme une chose qu'ils font et ont à faire ensemble alors même qu'ils sont en désaccord sur les principes de justice qui doivent le régir. Le compromis social-démocrate qui s'est élaboré en Europe occidentale dans les années d'après-guerre en est un bon exemple. Et si ce compromis est aujourd'hui mis à mal dans certains pays occidentaux, c'est notamment parce qu'il n'a pu s'ouvrir aux nouvelles formes que pourrait prendre la participation à la vie sociale ainsi qu'aux nouvelles formes d'exclusion socio-économique générées par les transformations du marché.

$2^{\circ} \mathrm{O} \mathrm{n}$ doit certes admettre que la conscience de la coopération entre les citoyens en vue d'un agir commun a très souvent émergé dans un mouvement d'opposition à un Autre présenté comme un ennemi commun. La guerre fut un ferment considérable dans la formation des identités politiques. M ais il n'est pas pour autant évident qu'il faille suivre Carl Schmitt lorsqu'il affirmait que la communauté politique ne peut se construire que sur la séparation ami/ennemi. Tout d'abord, parce que toute forme de coopération entre les citoyens doit être soumise à l'épreuve de la délibération publique. Si un projet implique toujours une part de lutte, voire l'exclusion (par exemple, sous la forme d'une discrimination entre nationaux et étrangers), celles-ci doivent être justifiables au regard de l'exigence universelle de l'égal respect. Ensuite, même si on peut admettre que tout dessein collectif ne peut que se construire dans une lutte pour la reconnaissance, nous sommes en mesure aujourd'hui d'imaginer une désubjectivation de cette lutte. Face aux défis politiques contemporains - la qualité de l'environnement, les menaces que font peser les intégrismes, la réduction de la dette,...- il ne s'agit plus tant pour une communauté humaine de s'opposer à une autre communauté, ni pour une classe d'en combattre une autre, mais plutôt de s'opposer à des forces intérieures qui habitent les individus et les sociétés et qui induisent des normes de reconnaissance injustes. 


\section{4 - Philosophiques / Automne 2002}

\section{5}

N ous venons de montrer que la démocratie délibérative présuppose structurellement l'instauration de formes sociales de reconnaissance prédiscursives. Je voudrais, pour terminer, évoquer un second problème que pose l'effectuation motivationnelle de l'idéal délibératif. La disposition à entrer dans la délibération publique ne repose pas seulement sur des conditions structurelles : la jouissance des droits fondamentaux et l'inclusion dans la coopération sociale. Elle suppose aussi que les discours produits dans l'espace public n'inhibent pas la participation de certains citoyens et ne handicapent pas la reconnaissance de la pertinence des jugements qu'ils posent. O r des paroles prononcées dans un débat public peuvent parfois être ressenties comme humiliantes ou blessantes. Lorsque cette blessure atteint le respect de soi ou l'estime de soi, elle peut inciter le citoyen à se tenir en marge du débat public, ou, pire encore, à être marginalisé par ses interlocuteurs. O n pense bien sûr à l'expression de propos racistes affirmant la supériorité d'une race ou d'une culture sur une autre. $M$ ais ce n'est peut-être pas ce type de propos qui pose les difficultés les plus importantes pour la réflexion qui nous occupe. Car on peut sérieusement mettre en doute le fait que de tels propos constituent des arguments et aient par conséquent une place légitime dans le débat public ${ }^{48}$. Une difficulté plus sérieuse est posée par des arguments qui, tout en étant de vrais arguments avancés et reproduits de manière sincère et réfléchie, peuvent aussi être blessants au point d'affecter le respect de soi de certaines personnes ou d'induire une image dépréciative de leur groupe d'appartenance.

C'est ainsi que dans nos sociétés contemporaines, certains dénis de reconnaissance peuvent résulter de la négation de la capacité de certaines personnes à accéder à l'autonomie en raison même de leurs pratiques ou de leurs appartenances religieuses ou culturelles. Le dénigrement et la dévalorisation de certaines identités culturelles ne repose plus ici sur l'affirmation de la supériorité d'un corpus de valeurs substantielles sur un autre, d'une conception de la vie bonne sur une autre (la supériorité des valeurs de l'O ccident chrétien sur celles d'autre cultures, par exemple). Une source plus insidieuse de dénigrement peut trouver son origine dans l'idée que certaines identités culturelles ne permettent pas (ou pas encore) cette forme de réflexivité critique qu'a apportée la culture moderne. C'est en raison même de ce déficit d'autonomie que l'on devrait cultiver une prudence vis-à-vis de certaines manifestations culturelles. M ême si l'idée est vraie ou en tout cas argumentée, son expression peut néanmoins être blessante.

Le débat sur le foulard islamique illustre très bien ceci. D ans cette controverse, une des positions communément avancées consiste à dire que le port du hijâb dans l'espace public est inacceptable parce qu'il est la manifestation d'un attachement à une tradition qui proscrit la distance critique

48. Ceci ne signifie pas nécessairement que la loi doit sanctionner l'expression de propos racistes. C'est un autre débat. 
et qui reproduit la domination masculine ${ }^{49}$. Ce type d'argument soulève en fait deux questions : celle de sa validité bien entendu (a) mais aussi celle des effets que peut produire son invocation en particulier dans certains contextes où se développe l'islamophobie (b).

(a) La validité de l'argument mentionné est discutable. Certains travaux sociologiques ${ }^{50}$ tendent à invalider l'interprétation qu'il donne du port du voile. Nombre de jeunes filles musulmanes qui portent le foulard le feraient par choix raisonné et la justification qu'elles apportent témoigne d'un degré de maturité et d'autonomie dans le jugement qui n'a rien à envier à celui des autres jeunes de leur âge. C'est ainsi que le port du foulard est pour certaines d'entre elles un moyen d'assurer leur promotion sociale par les études et par l'emploi tout en évitant une rupture avec leur famille. Bien entendu, ces observations n'excluent pas que dans d'autres situations, le foulard puisse être un moyen d'oppression. $M$ ais quoiqu'il en soit sur le fond, pour un défenseur de la démocratie délibérative, la question de la validité des arguments qui plaident pour ou contre le port du voile dans l'espace public ne peut, il est vrai, être tranchée a priori. Elle ne peut l'être que dans un débat public qui doit bien entendu associer les premières intéressées.

(b) N éanmoins si l'on veut associer pleinement les premières intéressées à un tel débat, il faut veiller à ce que l'expression publique de leur position ait un sens pour elle et qu'elles soient reconnues comme des personnes capables de poser un jugement sur leur propre pratique religieuse comme femme musulmane. Or c'est précisément ici quel'invocation de l'argument mentionné plus haut peut poser problème. Car indépendamment de la question de sa validité, l'expression, fut-elle prudente et bien intentionnée, de cet argument peut avoir des effets négatifs sur la reconnaissance des jeunes femmes qui sont visées et potentiellement sur leur possibilité effective de prendre part au débat public. Les études sociologiques mentionnées plus haut relèvent également que l'imputation d'absence de maturité, c'est-à-dire d'absence d'autonomie dans le jugement, qui leur est explicitement adressée peut être ressentie douloureusement, comme déni de reconnaissance, manque de respect par les jeunes femmes musulmanes qui en sont victimes ${ }^{51}$. O n ne mesure certainement pas assez - et on concédera que ce n'est pas aisé - l'impact que peut avoir ce type d'argument (reproduit dans d'autres contextes) sur celles ou ceux qui sont visés. Cet impact peut affecter directement

49. C'est en tout cas un point qui ressort clairement des analyses de la controverse, telle qu'elle s'est déroulée en Belgique et ailleurs. C'est ce que relève notamment J.-Y. Carlier, 2000, p. 247) : «Tel fut bien, chez nous aussi, l'enjeu pour beaucoup. Ces jeunes filles, revendiquant le port du foulard, n'étaient-elles pas manipulées par des intégristes décidés à remettre fondamentalement en question les acquis de la laïcité».

50. Brion, 2000.

51. Brion, 2000. 
le respect de soi et l'estime de soi, ou de manière indirecte contribuer à la reproduction d'une image sociale dépréciative d'un groupe ou d'une culture. Cet impact devient éminemment problématique pour la démocratie délibérative lorsqu'il conduit les victimes à ne pas s'exprimer publiquement («A quoi bon puisque nous n'en sommes pas capables!») ou, quand elles le font, à voir leur position disqualifiée sous prétexte de carence réflexive.

Q uelle conclusion doit être tirée du constat qui précède? II nous invite certainement à la modestie quant aux vertus de la pratique délibérative. Comme cela a été souligné plus haut (2.4), il est vraisemblable que cette pratique ait des vertus inductrices de reconnaissance et donc de respect de soi et d'estime de soi. M ais il est tout aussi vrai que dans certaines circonstances, l'expression publique de raisons peut générer de la mésestime qui à son tour inhibe et handicape la participation au débat public. Ce problème n'a vraisemblablement pas de réponse sociale structurelle. On perçoit mal en effet comment on pourrait réguler l'usage de la parole, a fortiori de la parole argumentée, de manière à éviter qu'elle ne soit blessante. Et même si c'était possible, imposer des limites substantielles à l'usage public de la raison serait à la fois paternaliste et contre-productif au regard de la promotion de l'idéal délibératif. $\mathrm{N}$ éanmoins le fait que l'expression de certaines positions puisse miner la reconnaissance mutuelle que suppose le débat public devrait nous inciter à une certaine prudence morale. Cette prudence pourrait s'exprimer sous la forme de la maxime suivante : même lorsque des arguments nous apparaissent pleinement justifiés, nous devons éviter que leur énonciation dans le débat public n'induise des effets de mésestime sociale qui empêcheraient certaines personnes de pouvoir exprimer leur point de vue ou de pouvoir le faire entendre pleinement dans la suite du débat. II faut surtout manier avec précaution les arguments ad hominem qui mettent en question les aptitudes de certaines personnes à poser des jugements, à argumenter ou à contribuer à la vie sociale. La maxime mentionnée ne justifie en aucun cas une quelconque limitation juridique à la liberté d'expression. $\mathrm{M}$ ais elle attire plutôt notre attention sur ce que J. Rawls aurait appelé un «devoir de civilité» 52 du citoyen lorsqu'il argumente dans l'espace public et qui ne peut se justifier qu'au nom même de la poursuite d'une pratique délibérative associant tous les intéressés.

\section{Concluons}

N otre parcours a d'abord visé à montrer qu'une compréhension adéquate del'enjeu normatif sous-jacent aux luttes pour la reconnaissance ne pouvait être honoré que par une forme délibérative de démocratie. Celle-ci permet

52. Rawls, 1993, p. 217. On notera que ce devoir est beaucoup moins contraignant que celui que propose Rawls quand il demande aux citoyens de sociétés pluralistes de s'abstenir d'invoquer tout argument qui présupposerait la vérité d'une doctrine compréhensive particulière. 
en effet une mise en question des normes de reconnaissance génératrices de mésestime sociale et peut, par les opportunités de participation politique qu'elle offre, générer du respect de soi et de l'estime de soi. Toutefois la disposition à participer à la délibération ne peut se développer pleinement que moyennant des formes sociales de reconnaissance qui ne se nouent pas exclusivement dans l'espace de la délibération. N ous avons essayé de tirer deux conséquences de cette thèse. Tout d'abord, la promotion de l'idéal délibératif repose sur des conditions structurelles, en particulier l'instauration d'une juste division sociale du travail. Ensuite, l'usage public de la raison dans la délibération doit demeurer attentif aux effets de l'énonciation de positions susceptibles de contribuer à la reproduction de formes de mésestime sociale qui, en retour, compromettraient la participation à la délibération.

\section{Bibliographie}

Brion, Fabienne, «D es jeunes filles à sauver aux jeunes filles à mater : identité sociale et islamophobie», dans M anço U., éd., Voix et voies musulmanes en Belgique, Bruxelles, Publications des Facultés universitaires Saint Louis, 2000, p. 115-146.

Dejours, Christophe, Souffrance en France. La banalisation de l'injustice sociale, Paris, Seuil, 1998.

Carlier, Jean-Yves, «D eux facettes des relations entre le droit et l'islam : la répudiation et le foulard», dans Dassetto $F$. (éd.), Facettes de l'islam belge, Bruxelles, Academia-Bruylandt, 2000, p. 239-251.

Castel, R obert, Les métamorphoses de la question sociale. Une chronique du salariat, Paris, Fayard, 1995.

Chaumont, Jean-M ichel et Pourtois, H ervé, éds, Souffrance sociale et attentes de reconnaissance. Autour du travail d'Axel Honneth, Louvain-laN euve, Recherches sociologiques, vol. 30, n², 1999.

Cohen, J oshua, «D eliberation and Democratic Legitimacy», dans H amlin, A. et Pettit, P. dir., The Good Polity. N ormative A nalysis of the State, Oxford, B. Blackwell, 1989, p. 17-34.

Cooke, $M$ aeve, "H abermas, A utonomy, and the Identity of the Self », Philosophy and Social Criticism, 18/3-4, 1992, p. 269-291.

Cooke, $M$ aeve, «Five arguments for deliberative democracy », Political Studies, vol. $48 / n^{\circ} 5$, p. 947-969.

Duhamel, André - Weinstock, Daniel et Tremblay Luc B., éds., La démocratie délibérative en philosophie et en droit : enjeux et perspectives, M ontréal, Editions Thémis, 2001.

Fishkin, James S., The Voice of the People. Public O pinion and D emocracy, $\mathrm{N}$ ew H aven-London, Yale University Press, 1995.

Fraser, $\mathrm{N}$ ancy, Justice Interruptus. Critical R eflections on the Postsocialist Condition, N ewYork-Londres, Routledge, 1996. 
Fraser, $\mathrm{N}$ ancy, « Social justice in the age of identity politics : redistribution, recognition, and participation », dans The Tanner L ectures on H uman Values, vol. 19, Salt Lake City, University of Utah Press, 1998a.

Fraser, $\mathrm{N}$ ancy, «Penser la justice sociale : entre redistribution et revendications identitaires», Politiques et sociétés, vol. 17, n³, 1998b, p. 9-37.

Fraser, $\mathrm{N}$ ancy, Adding Insult to Injury : Social Justice and the Politics of Recognition, Londres, Verso, 1999.

Fraser, $N$ ancy, «R ethinking recognition », $N$ ew Left Review, 3, may/june 2000, p. 107-120.

Gutmann, A my et Thompson, Dennis, Democracy and D isagreement, Cambridge (M ass.), H arvard University Press, 1997.

$\mathrm{H}$ abermas, J ürgen, Faktizität und $\mathrm{G}$ eltung. Beiträge zur D iskurstheorie des Rechts und des demokratischen Rechtsstaats, Frankfurt a. M., Suhrkamp. 1992, Tr. fr. : D roit et démocratie. Entre faits et normes, Paris, Gallimard, 1997.

H abermas, Jürgen, La pensée postmétaphysique. Essais philosophiques (trad. R. R ochlitz), Paris, A rmand Colin (Théories), 1993.

$H$ abermas, Jürgen, L'intégration républicaine : essais de théorie politique, Paris, Fayard, 1998.

H onneth, Axel, «Reconnaissance», dans Canto-Sperber, M onique, dir., Dictionnaire d'éthique et de philosophie morale, Paris, P.U.F., 1996, pp. 1272-1278.

H onneth, Axel, «Intégrité et mépris. Principes d'une morale de la reconnaissance», dans Chaumont J.-M . et Pourtois $H$., dir., Souffrance sociale et attentes de reconnaissance. A utour du travail d'Axel $\mathrm{H}$ onneth, Recherches sociologiques (Louvain-la-N euve), vol 30, n², 1999a, p. 11-22.

$\mathrm{H}$ onneth, Axel, «La démocratie, coopération réflexive : John Dewey et la théorie contemporaine de la démocratie», M ouvements, nº, 1999b, p. 169-178.

Kymlicka, Will, Liberalism, Community and Culture, , Oxford, Oxford University Press, 1989.

Kymlicka, Will, Contemporary Political Philosophy. An Introduction, Oxford, Clarendon Press, 1990.

Kymlicka, Will, M ulticultural Citizenship, Oxford, O xford University Press. 1995.

M argalit, Avishai, The D ecent Society (trans. N . Goldblum), Cambridge (M ass.), Harvard University Press, 1996.

M esure, Sylvie et R enaut, A lain, Alter ego. Les paradoxes de l'identité démocratique, Paris, Aubier, 1999.

Pamental, $M$ atthew, «W hat is it like to be a deliberative democrat», dans Tozer, Steve, dir., Philosophy of Education, Urbana, Philosophy of Education society, 1999. 
Pourtois, $H$ ervé, «D roits fondamentaux et démocratie délibérative», dans $M$. Coutu et al., dir., D roits fondamentaux et citoyenneté. Une citoyenneté fragmentée, limitée, illusoire?, M ontréal, Thémis; O nãti, Editions de I'IISJ , 2000, p. 55-86.

Sainsaulieu, R enaud, L'identité au travail, Paris, FN SP, 1987.

Shklar, Judith, «J ustice et citoyenneté», dans A ffichard, J. et de Foucauld J .B., Pluralisme et équité. La justice sociale dans les démocraties, Paris, Ed. Esprit, 1995, p. 85-101.

Taylor, Charles, Multiculturalism and the Politics of Recognition, Princeton, Princeton University Press. 1992, Tr. fr. : M ulticulturalisme. D ifférence et démocratie, Paris, Aubier, 1994.

Todorov, Tzvetan, «D u culte de la différence à la sacralisation de la victime», Esprit, $n^{\circ} 212,1995$, p. 90-102.

Tully, James, "Struggles over recognition and distribution », Constellations, 7/4, 2000, p. 469-482.

Vielle, Pascale, "Pour une "normalisation de la prostitution" », D ocuments de la Chaire H oover d'éthique économique et sociale, 77, Louvain-laN euve, Université catholique de Louvain, 2001.

Walzer, M ichael, «The Civil Society Argument», in Dimensions of Radical D emocracy : Pluralism, Citizenship, Community (Chantal, M offe, éd.), London, Verso, 1992, pp. 89-107.

Weinstock, Daniel, «Démocratie et délibération», Archives de philosophie, $63 / 3,2000$, p.405-421.

Young, Iris M arion, J ustice and the Politics of D ifférence, Princeton, Princeton University Press, 1990.

Young, Iris $\mathrm{M}$ arion, «Communication and the $\mathrm{O}$ ther : Beyond Deliberative Democracy », dans S. Benhabib, dir., D emocracy and Difference,

Princeton, Princeton University Press, 1996, pp. 120-135.

Young, Iris M arion (1997), « Unruly categories : a critique of N ancy Fraser's dual systems theory», N ew L eft Review, 222, p. 147-159. 